\title{
Design of the Multi-Agent Robot Group Formation Based on Pilot Followers
}

\author{
Haiyang Zou ${ }^{1}$ \\ ${ }^{1}$ Department of Computer College, China West Normal University, NanChong, SiChuan, China \\ Correspondence: Haiyang Zou, Department of Computer College, China West Normal University, China. E-mail: \\ haiyangzou@126.com
}

Received: November 27, 2015

Accepted: December 20, 2015

Online Published: January 6, 2016

doi:10.5539/cis.v9n1p70

URL: http://dx.doi.org/10.5539/cis.v9n1p70

\begin{abstract}
Some animals always keep a certain formation in flight, it plays a good role for the defense of animal predators, avoid falling behind, avoid obstacles and other aspects. Based on the the design of pilot follows formation way, this topic from the characteristics of bionics, which makes the robot population to maintain a certain formation, they complete the relatively complex tasks through mutual coordination, division and cooperation. We adopt asymmetric formation control strategy and introduce the graph theory in the topic, it has good theoretical and practical guiding significance about the application of robot in real.In the formation of scale design, We adopt hypercube structured design concept, and ant colony algorithm of sub-cube for hypercube secondary division, multi-agent robot groups has better results in all aspects of the expansion, fault tolerance and transaction complexity.
\end{abstract}

Keywords: formation way, multi-intelligence, figure, hypercube, ACO sub cube

\section{General Description of Multi-Agent Robot Population}

Throughout the history of human development, in order to survive, people can only make up a large and small tribe, through cooperation and common life, to resist the dangers of nature.With the rapid development of society, material and cultural life is constantly enriched, to take us convenience, the community also inevitable evolution. It must be huge, as a member of society, our capacity is very limited, in order to adapt to changes in society, is only human team-based, and constantly expand the scale of co-operation and take advantage of the intelligence community groups, to complete complex tasks. Therefore, teamwork has become an inevitable trend of social development. Similarly, the ideas of this cooperation group in many animal populations have also been reflected in a corresponding, for example, some of the creatures in nature, they do not have the intelligence of an individual, but through cooperation exhibit intelligent behavior, such as animal predation, nesting, to resist the invasion of the outside world. We called this biological set of decency to multi-agent groups, and formation is often groups of animals to take an effective behavior, they keep the certain geometric graphics or maintain a fixed position in the group, in order to complete the individual could not complete the complex work.In the design of multi-agent robot population, we can refer to the team concept of such cooperation.In the actual life, the individual robot is regarded as the individual in the multi agent, the individual is connected by direct or indirect communication, multi intelligent robot groups to accomplish more complex tasks.

Multi robot groups have those advantages in real life:

(1) in the design, we use a simple robot individuals, through cooperation and mutual coordination to complete more complex work, which is much simpler than the design complexity of a single robot; (2)In the event of unexpected events, the multi robot group is more adapt to the environment than a single robot for varied and complex work environment; (3) due to the use of distributed control system in the multi robot group, whether in time or in space, it is easier to achieve our demands; (4) Multi-intelligent robot population has better parallelism, redundancy, flexibility, robustness and fault tolerance.

The more of robots' number, could fulfill the complexity of task, the less time is required, if there is a faulty robot, especially the leader robot, it will lead to loss of control over the entire multi agent robot group, in order to solve this problem, we introduced the concept of the hypercube, hypercube is a topological structure, with highly redundant communication paths and strong fault tolerant communication ability.In the architecture, even though 
a third of the machine fails, the entire system can continue to complete the related tasks.

Based on all the advantages of multi-agent robots, it has been applied in many fields, such as: industry, agriculture, aviation and spaceflight, disaster relief and so on.

\section{Based on the Design of Leader Followers Formation}

\subsection{Control Strategy for Robots}

In order to achieve the unity of the robot groups, they must have a common strategy, but with the increase in the number of robots, the growth of information will show a terrible trend.In combination with the practice, we should adopt the distributed control mode in the design, the individual in the robot group do not need to obtain all the information, only need to obtain the information of several robots, which can significantly reduce the system's requirements for communication and improve the system's flexibility.

\subsection{Formation Design}

The reason which we choose the way of formation, people from the bionics to get inspiration, some animal flight to maintain a specific formation, which is the defense of predators, to avoid falling behind, avoid obstacles and other aspects have played a good role. Based on the follow of leader information design, the robots maintain a certain formation, through cooperation and coordination to complete more complex work.

\subsection{Leaders and Followers}

Follow the leader in multi-agent group, we choose a leader as having leadership function, the other agent are as a follower, can only be by the leader; in the process, it is particularly important to keep a certain formation and cooperation, collision avoidance and obstacle avoidance are necessary for us to solve the problem.Collision avoidance is to prevent collisions between groups of multi intelligent robot; obstacle avoidance is when there is obstruction, the robots can successfully avoided and maintained previously formation set to continue to the unfinished task.

\subsection{Graph Theory}

In order to express the relationship between the multi robot groups, we introduce the theory of graph.

Definition 1: We often use a tuple $(V, E)$ to represent a graph, elements $V$ are called vertices and nodes, $|V|$

is number of vertices in the graph, $\mathrm{E}=\left\{e_{1}, e_{2}, e_{3} \ldots e_{m}\right\}$, elements in $\mathrm{E}$ representing edges, $e_{i}$ is $\left(V_{i}, V_{j}\right), e_{i}$ is

the edge of $V_{i}$ and $V_{j}$.

We regard robot as the point in the graph, the communication link between robots as edge of the graph.How to determine the edges of the graph, so that the edge of the information chain can deliver information at least, to achieve our requirements.In the formation process of multi agent, we apply the theory of rigid figure.

Definition 2: Figure of rigidity: if any one of the edges is removed, the graph is not rigid, we call this graph is the smallest rigid graph.

How to judge whether a graph is rigid, we judge by $\operatorname{Laman}^{[2]}$ :

Definition 3: Let $G(V, E)$ is a graph in the two-dimensional plane, $|V|$ is vertex, $|E|$ is edge, $G$ is rigid, there

is a subset $E^{\prime}, E^{\prime} \subseteq E$, it must meet the following two conditions.

(1) $\left|E^{\prime}\right|=2|V|-3$

(2) $E^{\prime \prime} \subseteq E^{\prime}, E^{\prime \prime} \neq \phi,\left|E^{\prime \prime}\right| \leq 2\left|V\left(E^{\prime \prime}\right)\right|-3$,

\subsection{Design of Hypercube}

In multi-agent robot group, the number of robots, the complexity of the task can be completed more highly, the 
less time required.But with the increasing number of robots, the communication cost of the system will be increased by the explosive growth. Once there is a failure of robot, it is possible to cause the failure of the task. How to solve this problem, we can introduce the concept of the formation hypercube design.

The topological structure of the hypercube has the following features:

(1) in robots group, it should include $2^{n}$ robots and $n * 2^{n-1}$ communication lines; (2) the number of robots in robots group is a special number for the length of $n$ by binary; (3) there is only one difference between the number of adjacent robots, and all robots must be able to communicate each other;

We use the concept of the hypercube, the super large scale multi robot group to carry out the formation, the key problem is solved for fault tolerance massive growth of data generated, communication efficiency, communication overhead and so these three areas.

\subsection{Ant colony Sub Cube}

In the design of hypercube, we require every robot to communicate with each other, there are $n$ robots, then it requires the existence of communication channel between them, but this will lead to a huge increase in the amount of communication, with a sharp increase in traffic, make hardware for conveying data to failure, which ultimately lead to the failure task.

At first, we divided hypercube structure for large scale robots in the algorithm, only the leader robots for hypercube formation, followed by the use of based on ant colony sub cube algorithm for the second division, layer by layer to achieve hypercube structure, reduce the amount of information between robots groups, greatly improve the communication efficiency. In this way, we can ensure that the system has a certain number of robots, but also the characteristics of the super cube algorithm.

\section{Algorithm Design}

\subsection{The Design of Collision Avoidance Algorithm}

In the design, the robot groups maintain a certain distance, the distance is only in a reasonable range, the distance is too large, the communication between agents is easily affected, it is difficult to keep the formation; the distance is too small, and easy to produce collisions between robots.

In the intelligent community, we assume that there are $\mathrm{N}$ robots, one leader, the rest of the N-1 is followers:

$$
\begin{gathered}
q_{i}(t)=p_{i}(t) \\
p_{i}(t)=u_{i}(t) \quad i=1,2, \cdots N
\end{gathered}
$$

$q_{i}(t), p_{i}(t) \in R^{N}$ is the robot's position and speed, we will communicate combination between leader and followers into a collection.

$$
M_{i}(t)=\left\{j:\left\|q_{i}-q_{j}\right\|<r, j=2, \cdots N, j \neq i\right\}
$$

$\|\cdot\|$ is the Euclidean norm, $r$ is the radius of the intelligent robot can perceive, if there is a communication between the robot, when $\left\|q_{i}-q_{j}\right\|<r$, Only adjust the speed and angle of the followers, when $\left\|q_{i}-q_{j}\right\|>r$, not only adjust to follows the angle and speed, that is, to accelerate the followers of speeding, but also slow down the leader of speeding until it reaches the best results.

\subsection{Design of Obstacle Avoidance Algorithm}

When the robots community meets various size of a barrier, we can simulate the obstacle to become the radius of

$\mathrm{R}\left(X_{i}, Y_{j}\right)$ to round, The vertical $L_{i}$ node $\left(X_{m}, Y_{n}\right)$ is to the obstacle for the shortest distance:

$$
D=\sqrt{\left(X_{i}-X_{m}\right)^{2}+\left(Y_{j}-Y_{m}\right)^{2}}-R_{i}
$$

We should not only look for the shortest distance, but also make the robot can avoid obstacles. We can define the 
objective function:

$$
F=L_{K}+\delta \sum_{i=1}^{m-1} \frac{1}{D_{\min }}
$$

$L_{K}$ represents the path length of the $\mathrm{K}$ robot, $\mathrm{D}$ indicates the distance between the nodes and the obstacles, $\delta$ is avoidance factor, $D_{\min }$ is the shortest distance between each robot to obstacle.

\subsection{Algorithm Based on Ant Colony Algorithm for Sub Cube}

Based on ant colony sub cube is a complete NP problem, we can handle cube to combinatorial optimization problem, in algorithm design, based on ant colony algorithm, it is actually a large-scale hypercube into small sub cube structure.

$$
P_{i j}^{k}(t)=\left\{\begin{array}{c}
\frac{\left[\tau_{i j}(t)\right]^{\alpha}\left[\eta_{i j}\right]^{\beta}}{\sum_{s \in \text { allowed }_{k}}\left[\tau_{i s}(t)\right]^{\alpha}\left[\eta_{i s}\right]^{\beta}} \quad j \in \text { allowed }_{k} \\
0
\end{array}\right.
$$

$\operatorname{tabu}_{k}(k=1,2,3, \cdots n):$ A collection of all the nodes currently traversed by the ant $\mathrm{k}$, allowed $_{k}$ : a collection of no walking for ants.

$$
\begin{gathered}
\tau_{i j}(t+n)=(1-\rho) \cdot \tau_{i j}(t)+\Delta \tau_{i j} \\
\Delta \tau_{i j}=\sum_{k=1}^{m} \Delta \tau_{i j}{ }^{k}
\end{gathered}
$$

$\rho$ : Pheromone evaporation coefficient $(1-\rho)$ :Pheromone residual factor; $\Delta \tau_{i j}$ Pheromone increment in this cycle

$\Delta \tau_{i j}{ }^{k}$ : the amount of information left in the (i,j) of the cycle in this cycle for ant's $\mathrm{K}$

In the algorithm, the distribution density of the robot is heuristic information $\eta_{i j}, \tau_{i j}$ and $P_{i j}^{k}(t)$, is the sub cube, $\mathrm{j}$ is the dimension of division, $\eta_{i j}$ is the last of the two smallest communication path length of a robot capable of communicating in a hypercube, because the communication line is longer, the more cost of communication.

$$
\eta_{i j}=\frac{1}{\left(L_{1(J)}, \quad L_{2(J)}\right)}
$$

\section{Our Expectations and Shortcomings}

In the multi robot group formation, in order to maintain a fixed formation and the formation must be rigid, in order to achieve rapid real-time communication in the whole process, and the communication of information in the least amount of, we introduce the graph theory, and the application of Laman's theory, to determine whether a formation is rigid; in order to reduce the complexity of the control, in control strategy we adopt an asymmetric formation of distributed control strategy; for multi-agent robots group excessive number of machines in the group, due to traffic too much, in the event of a hardware failure, thus affecting the entire multi-agent robot control. In order to avoid this, we have been inspired by the principle of the hypercube.In order to further improve the system's expansion, fault tolerance, the processing degree of complex things. But some defects still exist in the algorithm: (1) the algorithm due to the existence of multiple cycle and iterative process, when super 
cube dimension is large, algorithm of real-time performance is not very ideal, it could appear overhead time of the phenomenon; (2) in the collision avoidance algorithm doesn't make external environmental factors into consideration; (3) the formation algorithm lack of certain adapting, in the changing environment, we should not adopt fixed formation, but should be according to the actual situation to choose the best formation for the multi-agent by themselves.

\section{References}

Laman, G. (1970). On graphs and rigidity of plane skeletal structures. Journal of Engineering Mathematics, 4(4), 331-340. http://dx.doi.org/10.1007/BF01534980

Wang, H. W. (2013). Research on multicast routing optimizing strategy in hyoercube (doctoral dissertation). Harbin Institute of Technology. Retrieved from http://d.g.wanfangdata.com.cn/Thesis_D421187.aspx

Wang, X. K., Li, X., \& Zheng, Zh. Q. (2013). survey of developments on multi-agentFormation control related problems. Control and decision making, 28(11), 1601-1608. Retrieved from http://d.g.wanfangdata.com.cn/Periodical_kzyjc201311001.aspx

Wang, Y. (2012). Research on formation control merhods for multi-agent (master's thesis). Harbin Institute of Technology. Retrieved from http://d.g.wanfangdata.com.cn/Thesis_D240753.aspx

Wu, Q. D., \& Wang, L. (2004). Intelligent ant colony algorithm and application. Shanghai, SH:science and Technology Education Press. Retrieved from http://hn.sslibrary.com/book/card?cnFenlei=TP273\&ssid=11402640\&d=3eb7d91fed1486a73b2e01499eabb $7 \mathrm{ca} \& \mathrm{dxid}=000005031709 \&$ isFromBW $=$ false $\&$ isjgptjs $=$ false

Zhang, Sh. (2012). Formation control robustness analysis of mobile multi-agent system (doctoral dissertation). Master Thesis of $\mathrm{Lu}$ dong University. Retrieved from http://210.41.203.155/kcms/detail/detail.aspx?recid=\&FileName=1012403929.nh\&DbName=CMFDLAST 2012\&DbCode=CMFD\&uid=aXdoWmlVbFJGMHV1M2FadHNDbXJ6Si9HVW1BN0ttS0QvOExrQzNqT DVNM2ZqcFZL

\section{Copyrights}

Copyright for this article is retained by the author(s), with first publication rights granted to the journal.

This is an open-access article distributed under the terms and conditions of the Creative Commons Attribution license (http://creativecommons.org/licenses/by/3.0/). 